\title{
Global monopole, dark matter and scalar tensor theory
}

\author{
F.Rahaman*, M.Kalam ${ }^{\ddagger}$ R.Mondal *and B.Raychaudhuri ${ }^{\dagger}$
}

\begin{abstract}
In this article, we discuss the space-time of a global monopole field as a candidate for galactic dark matter in the context of scalar tensor theory.
\end{abstract}

It is believed that our Universe in the past was denser and hotter. So the early stage of the Universe was in a systematic phase and there were no topological defects. As the Universe expands, it cools down from its hot initial state [1-3]. So the early Universe had undergone a number of phase transitions. Phase transitions in the early Universe can give rise to various forms of topological defects. They can be monopoles, comic strings or domain walls. Among them monopoles and cosmic string are well studied for their cosmological as well as astrophysical implications. Monopoles are point like topological defects that may arise during phase transitions in the early universe. There has been a fairly large amount of discussions [4-24] on the gravitational field of global monopoles beginning with the work of Barriola and Vilenkin (BV) [25]. According to BV, global monopoles are the configurations with energy density decreasing with the distance as $\frac{1}{r^{2}}$. Recent observations indicate that the galaxies, cluster of galaxies and super clusters are filled out with 90 percent of dark matter [26]. Rubin et al [27] have found that the rotation curve profile of a spiral galaxy is flat outside a central galactic region and they have suggested the energy density varies as $\frac{1}{r^{2}}$ of non luminosity matter ( dark matter ) which should contribute to the flatness of the rotation curves [28]. Nucamendi and others [29-31] have suggested that the monopole ( its energy density proportional to $\frac{1}{r^{2}}$ ) could be the galactic dark matter in the spiral galaxies. In a recent work, Lee and Lee [32] studied global monopole field ( within weak field approximations ) as a candidate for galactic dark matter in the scalar tensor theory of gravity inspired by the work of Nucamendi. The measurements, made by Persic et al [33] of rotation curves in spiral galaxies show that the coplanar orbital motion of gas in the outer parts of these galaxies keeps more or less a constant value up to several luminous radii.

\footnotetext{
${ }^{0}$ Pacs Nos : 04.20 Gz,04.50 + h, 04.20 Jb

Key words: Global monopole, Scalar tensor theory, Dark matter

*Dept.of Mathematics, Jadavpur University, Kolkata-700 032, India E-Mail:farook_rahaman@yahoo.com $\ddagger$ Dept. of Phys. , Netaji Nagar College for Women, Regent Estate, Kolkata-700092, India.

$\dagger$ Dept. of Phys. , Surya Sen Mahavidyalaya, Siliguri, West Bengal, India
} 
The renewed interest in the scalar theory of gravitation is mainly due to the belief that, at least at sufficient high energy scale, gravity becomes scalar tensorial in nature and these theories are important in the very early Universe [34-35]. In this article, we investigate the space-time of a global monopole field as a candidate for galactic dark matter in the context of scalar tensor theory. The gravitational field equations for a global monopole in scalar tensor theory written in Dicke's revised units [36], in general

$$
G_{a b}=-T_{a b}-\frac{1}{2} \phi^{-2}(2 \omega+3)\left[\phi_{, a} \phi_{, b}-g_{a b} \phi_{, \alpha} \phi^{, \alpha}\right]
$$

where $T_{a b}$ is the energy momentum tensor due to the monopole field, $\phi$ is the Brans-Dicke scalar field and $\omega$ is the Brans-Dicke parameter. The energy momentum tensor of a static global monopole can be approximated ( outside the core) as [25]

$$
T_{t}^{t}=T_{r}^{r}=\frac{\eta^{2}}{r^{2}} ; T_{\theta}^{\theta}=T_{\phi}^{\phi}=0
$$

where $\eta$ is the energy scale of symmetry breaking . As a result of spherically symmetry, we consider $\phi=\phi(r)$ and line element

$$
d s^{2}=e^{\nu} d t^{2}-e^{\beta} d r^{2}-r^{2} d \Omega_{2}^{2}
$$

Substituting this in equation (1) and taking into account equation(2), we obtain the following set of equations

$$
\begin{gathered}
e^{-\beta}\left[\nu^{\prime \prime}+\frac{1}{2}\left(\nu^{\prime}\right)^{2}-\frac{1}{2} \nu^{\prime} \beta^{\prime}+\frac{\nu^{\prime}-\beta^{\prime}}{r}\right]=-\frac{k}{2}\left(\psi^{\prime}\right)^{2} e^{-\beta} \\
e^{-\beta}\left[\frac{1}{r^{2}}+\frac{\nu^{\prime}}{r}\right]-\frac{1}{r^{2}}=-\frac{\eta^{2}}{r^{2}}+\frac{k}{2}\left(\psi^{\prime}\right)^{2} e^{-\beta} \\
e^{-\beta}\left[\frac{1}{r^{2}}-\frac{\beta^{\prime}}{r}\right]-\frac{1}{r^{2}}=-\frac{\eta^{2}}{r^{2}}-\frac{k}{2}\left(\psi^{\prime}\right)^{2} e^{-\beta}
\end{gathered}
$$

[ where $\psi=\ln \phi$ and $k=\frac{1}{2}(2 \omega+3)$ and " refers to differentiation with respect to radial coordinate ]

The wave equation for the Brans-Dicke scalar field is given by

$$
\psi^{\prime \prime}+\frac{2}{r} \psi^{\prime}+\psi^{\prime}\left[\frac{\nu^{\prime}}{2}-\frac{\beta^{\prime}}{2}\right]=-\frac{e^{\beta} \eta^{2}}{k r^{2}}
$$

Matos et al [28-29] have examined the possibility and type of dark matter that determining the geometry of a space-time where the flat rotational curves could be explained. 
We are to consider global monopole in scalar tensor theory as a candidate for galactic dark matter. To discuss rotational curves in the galaxy, we consider the circular motion of stars in space-time with metric (3). Now the Lagrangian for a test particle traveling on this space-time described by (3) is

$$
2 L=e^{\nu}(\dot{t})^{2}-e^{\beta}(\dot{r})^{2}-r^{2}(\dot{\theta})^{2}-r^{2} \sin ^{2} \theta(\dot{\phi})^{2}
$$

[ where dot refers to differentiation with respect to proper time]

We consider the case, $\theta=\frac{\pi}{2}$. We get generalized momenta from (8) as

$$
\begin{gathered}
p_{t}=E=e^{\nu} \dot{t} \\
p_{r}=-e^{\beta} \dot{r} \\
p_{\phi}=-W=r^{2} \dot{\phi}
\end{gathered}
$$

where $\mathrm{E}$ is the total energy and $\mathrm{W}$ is angular momentum. Using (8),(9) and (11), the geodesic equations read

$$
(\dot{r})^{2}+V_{e f f}(r)=0
$$

where $V_{\text {eff }}(r)=e^{-\beta}\left[1+\frac{W^{2}}{r^{2}}-E^{2} e^{-\nu}\right]$. We require the following conditions for stars to have circular motions [37]

$$
\dot{r}=0, \frac{\partial V_{e f f}}{\partial r}=0, \frac{\partial^{2} V_{e f f}}{\partial r^{2}}>0
$$

Following [37], it is found that the tangential velocity of the test particle is

$$
v^{\text {tangential }}=v^{\phi}=\sqrt{ }\left[\frac{r\left(e^{\nu}\right)^{\prime}}{2 e^{\nu}}\right]
$$

[ where " refers to differentiation with respect to radial coordinate ]

It is easy to show that if flat rotation curves are required, it arises the following flat curve condition from (14) i.e.

$$
e^{\nu}=B_{0} r^{l}
$$

where $l$ is given by $l=2\left(v^{\phi}\right)^{2}$ and $B_{0}$ is an integration constant. The observed rotational curve profile in the dark matter dominant region is such that the rotational velocity $v^{\phi}$ more or less a constant. For a typical galaxy the rotational velocities are $v^{\phi} \sim 10^{-3}(300 \mathrm{~km} / \mathrm{s})[29]$. 




Figure 1: The diagram for $e^{\nu}$ ( taking $B_{0}=1$ and $v^{\phi} \sim 10^{-3}$ )

By (6) -(5) -2(4), one gets,

$$
\nu^{\prime \prime}+\frac{\left(\nu^{\prime}\right)^{2}}{2}+\frac{2 \nu^{\prime}}{r}=\frac{\beta^{\prime} \nu^{\prime}}{2}
$$

This implies

$$
e^{\nu}\left(\nu^{\prime}\right)^{2}=\frac{e^{\beta} b^{2}}{r^{4}}
$$

where b is an integration constant. From (17), by taking into account (15), we get

$$
e^{\beta}=\frac{B_{0} l^{2} r^{l+2}}{b^{2}}
$$

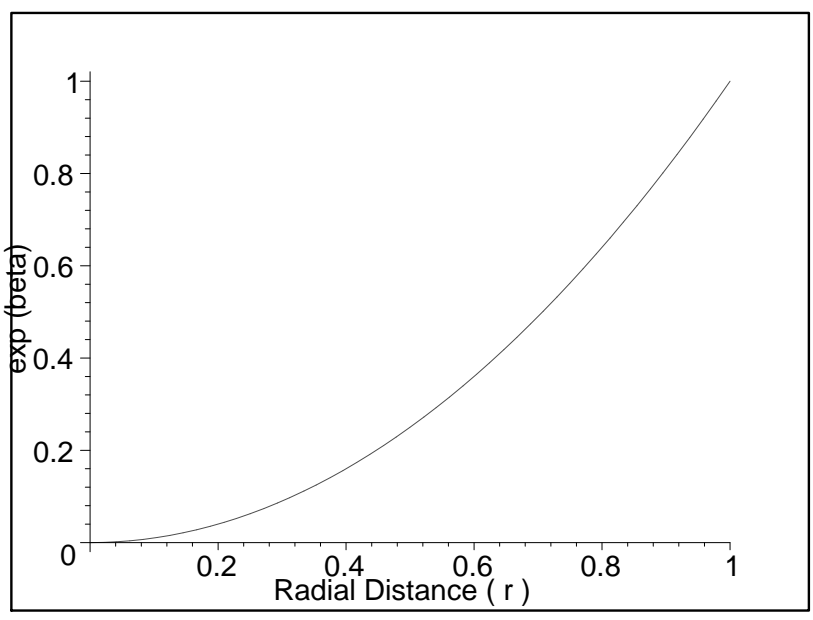

Figure 2: The diagram for $e^{\beta}\left(\right.$ taking $B_{0} l^{2}=b^{2}$ and $\left.v^{\phi} \sim 10^{-3}\right)$ 
Also from wave equation (7), by using (15) and (18), we get

$$
\psi=-\frac{B_{0} l^{2} r^{l+2} \eta^{2}}{k b^{2}(l+2)^{2}}+D \ln r+F
$$

where D and F are integration constants. One should note that actual Brans-Dicke scalar field

$$
\phi=e^{\psi}=r^{D} \exp \left[F-\frac{B_{0} l^{2} r^{l+2} \eta^{2}}{k b^{2}(l+2)^{2}}\right]
$$

If we take $\mathrm{D}$ and $\mathrm{F}$ to be zero, then

$$
\phi=\exp \left[-\frac{B_{0} l^{2} r^{l+2} \eta^{2}}{k b^{2}(l+2)^{2}}\right]
$$

Here one can see that if $\omega \rightarrow \infty, \phi \rightarrow 1$ and $\phi \rightarrow 0$ as $r \rightarrow \infty$.

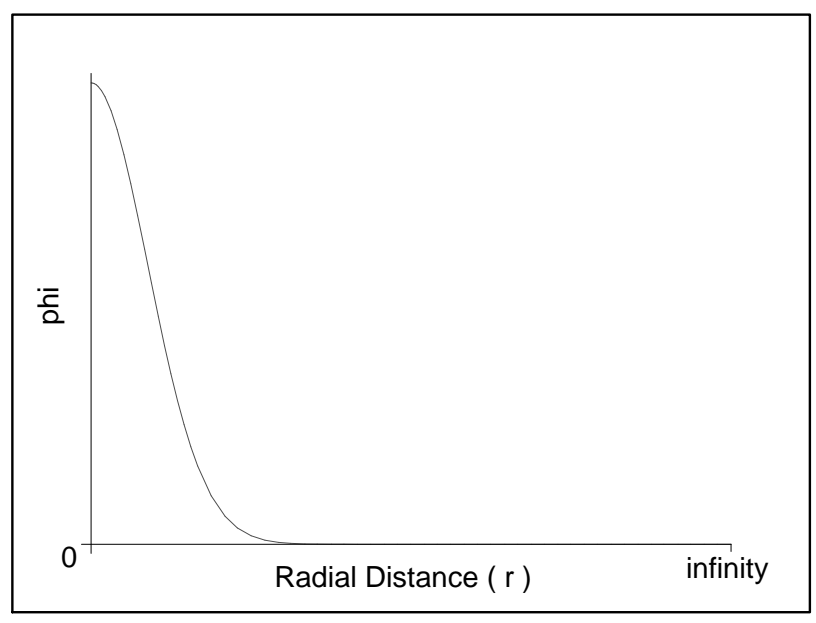

Figure 3: The diagram for Brans-Dicke scalar field $\phi\left(\right.$ taking $B_{0} l^{2} \eta^{2}=k b^{2}(l+2)^{2}$ and $v^{\phi} \sim 10^{-3}$ )

With the flat curve condition, metric (3) becomes

$$
d s^{2}=B_{0} r^{l} d t^{2}-\frac{B_{0} l^{2} r^{l+2}}{b^{2}} d r^{2}-r^{2} d \Omega_{2}^{2}
$$

This metric is analogous to the metric of general relativistic version of a matter distribution, where moving test particles follow a constant rotational curve. If we choose $R=\frac{l r^{2}}{2 b}$, then our metric takes the form ( except for a conformal factor)

$$
d s^{2}=d t^{2}-d R^{2}-\frac{1}{B_{0}}\left[\frac{2 b R}{l}\right]^{\frac{2-l}{2}} d \Omega_{2}^{2}
$$


From eq.(23), we see that the curved space-time of global monopole field as a candidate for galactic dark matter presents a deficit solid angle in the hypersurfaces $t=$ constant. The area of a sphere of radius $\mathrm{R}$ in this space would different from $4 \pi R^{2}$. Here the angular deficit is dependent on the rotational velocity of the galaxies. Galaxies are composed by almost 90 percent of dark matter distributed at the halos and for a typical galaxy, the rotational velocity more or less a constant suggests the existence of dark matter whose energy density varies as $\frac{1}{r^{2}}$. The energy density of global monopole proportional to $\frac{1}{r^{2}}$, so according to Nucamendi et al the monopole could be the galactic matter in the spiral galaxies. Assuming energy density proportional to $\frac{1}{r^{2}}$, we have found a spherically symmetric metric (22) with flat curve condition. It is important to note that our metric is not conformally flat and hence it represents a monopole. Pando, Valls and Gaboud [38] have proposed that topological defects are responsible for the structure formation of the galaxies. Our result is in agreement with Nucamendi that monopole could be the galactic dark matter in the spiral galaxies. Thus it seems monopoles ( one of the topological defects ) take part an important contribution of the galaxy formations and give indirect support of Pando et al proposal.

\section{Acknowledgements}

F.R is thankful to Jadavpur University and DST , Government of India for providing financial support under Potential Excellence and Young Scientist scheme. MK has been partially supported by UGC, Government of India under Minor Research Project scheme. We are grateful to the anonymous referee for his valuable comments and constructive suggestions. 


\section{References}

[1] Kibble, T.W.B. J. Phys. A 9, 1387(1976)

[2] A.Vilenkin and E.P.S. Shellard (1994), Cosmic String and other Topological Defects (Camb. Univ. Press)

[3] T Vachaspati arXiv: hep-th 0101270

[4] D.Harrari and C.Lousto Phys. Rev. D 42,2626(1990)

[5] X. Shi and X. Li Class. Quan. Grav. 8,761(1990)

[6] P.Breitenlohner, P.Forgacs and D.Maison Nucl.Phys,B383, 357(1992)

[7] A.Linde arXiv: astro-ph / 9402031

[8] A.Banerjee, S.Chatterjee and A.A.Sen Class. Quan. Grav. 13, 3141(1996)

[9] A.Banerjee, A . Beesham, S.Chatterjee and A.A.Sen Class. Quan. Grav. 15, 645(1998)

[10] I.Cho and A.Vilenkin Phys. Rev. D 56(1997) 7621

[11] S.Chakraborty Physica Scripta 58,294(1998)

[12] F Rahaman , P Ghosh, M Kalam and S Mandal Int. J. Mod.Phys.A 20, 993(2005)

[13] A.Barros and C.Romero Phys. Rev. D 56,6688(1997)

[14] O.Dando and R.Gregory arXiv: gr-qc/ 9709029

[15] S.Liebling arXiv: gr-qc/9906014

[16] S.Liebling arXiv: gr-qc/ 9904077

[17] E.R.B. de Mello Braz.J.Phys. 31,211(2001)

[18] R.M.T. Filho and V.B. Bezerra Phys. Rev.D 64,084009 (2001)

[19] K.A.Bronnikov, B.E.Meierovich and E.R. Podolyak arXiv: gr-qc/ 0212091

[20] E.R.B. de Mello arXiv: hep-th/ 0210236

[21] Li.X and Lu.J Phys.Rev.D 62, 107501(2000)

[22] X. Li and J. Hao arXiv: hep-th/ 0210058

[23] K.A.Bronnikov arXiv: gr-qc/ 0301084

[24] F Rahaman, S Sur and K Gayen Physica Scripta 69, 78 (2004) 
[25] M.Barriola and A.Vilenkin Phys. Rev. Lett. 63, 341(1989)

[26] Siddhartha.F Guzman, Matos.T, Nunez.D and Ramirez.E arXiv: astro-ph/0003105

[27] Rubin.V, Thonnard.N, Ford.W and Roberts.M ApJ 81, 719(1997)

[28] Matos.T, Siddhartha.F Guzman and Lopez.L arXiv: astro-ph/9908152

[29] Matos.T, Siddhartha.F Guzman and Lopez.L arXiv: astro-ph/0003398

[30] Nukamendi.U, Salgado.M and Surarsky.D Phys.Rev.Lett. 84, 3037(2000)

[31] Nukamendi.U, Salgado.M and Surarsky.D Phys.Rev.D 63, 125016(2001)

[32] Lee.T and Lee.B Phys.Rev.D 69, 127502 (2004)

[33] Persic.M, Saluci.P and Stel.F MNRAS 281, 27 (1997)

[34] Green.M, Schwarz.J and Witten.E Superstring Theory ( Cambridge University Press, 1987)

[35] Brans C and Dicke R.H Phys.Rev. 124, 925(1961)

[36] Dicke.R Phys.Rev. 125, 2163(1962)

[37] Chandrasekhar.S Mathematical theory of Black holes, Oxford Science Publications (1983)

[38] J.Pando, D.Valls-Gaboud and L.Fang Phys.Rev.Lett. 81,8568 (1998) 